\title{
Direct Self-Repairing Control for Quadrotor Helicopter Attitude Systems
}

\author{
Huiliao Yang, Bin Jiang, and Ke Zhang \\ College of Automation Engineering, Nanjing University of Aeronautics and Astronautics, Nanjing 210016, China \\ Correspondence should be addressed to Bin Jiang; ebjiang@yahoo.com
}

Received 12 December 2013; Accepted 28 February 2014; Published 31 March 2014

Academic Editor: Rongni Yang

Copyright (C) 2014 Huiliao Yang et al. This is an open access article distributed under the Creative Commons Attribution License, which permits unrestricted use, distribution, and reproduction in any medium, provided the original work is properly cited.

\begin{abstract}
A quadrotor helicopter with uncertain actuator faults, such as loss of effectiveness and lock-in-place, is studied in this paper. An adaptive fuzzy sliding mode controller based on direct self-repairing control is designed for such nonlinear system to track the desired output signal, when any actuator of this quadrotor helicopter is loss of effectiveness or stuck at some place. Moreover, using the Lyapunov stability theory, the stability of the whole system and the convergence of the tracking error can be guaranteed. Finally, the availability of the proposed method is verified by simulation on 3-DOF hover to ensure that the system performance under faulty conditions can be quickly recovered to its normal level. And this proposed method is also proved to be better than that of LQR through simulation.
\end{abstract}

\section{Introduction}

Quadrotor helicopter is one kind of electric VTOL. Compared with the conventional rotor helicopter, quadrotor can generate more lift force and its structure is more compact. Especially, its four rotors can counteract the reaction torque mutually, so the propellers against reaction torque are not needed [1]. Due to these properties, it makes quadrotor monitor and detect the targets close to the ground so that it has broader military and civilian prospect.

On the other hand, quadrotor, which is the underactuated system with 6-DOF and 4 outputs, has the properties of multivariety, nonlinearity, strong coupling, and sensitivity to disturbance. Once it has some faults, it may lead to the loss of performance of flight, even loss of control. Thus, selfrepairing control is born.

Self-repairing control, which utilizes the redundancy of the control system under normal working condition to improve the adaptability to the fault of the flight control system, can avoid catastrophes and make the faulty aircraft operate safely. Then, self-repairing control consists of the direct one and the indirect one. Direct self-repairing control does not need accurate system parameters, while system parameters and several control strategies are the necessity in indirect self-repairing control.
As is known to all, attitude control is the key point of the whole flight control. In addition, the attitude and position of quadrotor helicopter have the direct coupling. Therefore, the research on attitude controller with the capability of selfrepairing from fault is imperative.

Recently, research on the flight control of mini quadrotor helicopter has got some achievements. For instance, Bouabdallah from EPFL has developed several control methods, such as PID, LQR, and Backstepping, based on OS4 [2], one kind of mini quadrotor helicopter, and realized the control on attitude during flight. But Altug from University of Pennsylvania has designed the controller of the quadrotor helicopter HMX4 [3] based on Backstepping and, moreover, actualized the autonomous hover control with the help of vision orientation. Afterwards, vertical takeoff and landing of quadrotor helicopter based on neural network control were achieved by J. Dunfied. Then, Wang has focused on the robust control method based on $H_{\infty}$ [4], which can guarantee the tracking performance and noise immunity of quadrotor helicopter.

However, the existing works on fault diagnosis and faulttolerant control of quadrotor helicopter are quite few at present. A Backstepping fault-tolerant controller for quadrotor helicopter system based on the estimation of compound interference and partial FDI was proposed in [5]. And one 
kind of robust fault detection module with observer based on model was designed to reconfigure control law in [6]. In addition, a state estimator for fault detection is proposed in [7] to reconfigure the structure of controller for quadrotor so that it can recover some of control performances when a fault occurs. Yet there is still some weakness in the articles above; firstly, the models of quadrotor are obviously inaccurate. Then, the control strategies are somewhat complicated, which should contain fault identification; thus, it is difficult for application.

The need for effective and realizable fault-tolerant control for quadrotor helicopter with uncertain actuator faults motivates this research. In this paper, we develop an attitude system for quadrotor based on an adaptive fuzzy sliding mode tracking control to compensate the actuator fault such as loss of effectiveness and lock-in-place. The main contributions of this paper are as follows.

(1) The nonlinear model of quadrotor helicopter is put forward in detail, while the linear model ignoring the nonlinear factors such as gyroscopic effect is inaccurate when designing the fault-tolerant control system.

(2) An adaptive fuzzy sliding mode controller without fault identification is designed to track the desired output signal so that quadrotor can finish its mission safely even when any actuator of this quadrotor is loss of effectiveness or stuck at some place.

The rest of this paper is organized as follows. In Section 3.2 , the stability of the whole system is guaranteed by Lyapunov stable principle. In Section 4, the proposed method is verified by simulation on 3-DOF hover and also proved to be better than the LQR method which is often used in the attitude system of quadrotor. Finally, conclusions follow in Section 5 .

\section{Modeling Process}

2.1. System Model. The attitude and position of quadrotor helicopter are operated by the rotor's rotation rate, without the auto bank unit. The structure schematic is shown in Figure 1.

Three attitude angles are controlled in these principles shown in Figure 2: the roll moment is generated by the difference between the speed of right and left rotor so that the roll angle changes. In the same way, the pitch angle is controlled by the front and back rotor. While the yaw angle changes, the rotors in the diagonal rotate in the same speed and the speed of rotors in different diagonal differs.

To limit the complexity of the dynamics modeling, the following assumptions are adopted [8].

(1) The whole structure is rigid and symmetrical.

(2) Thrust and drag forces are proportional to the square of propellers speed rotation.

(3) The variable range of attitude angles is small (generally less than $5^{\circ}$ ).

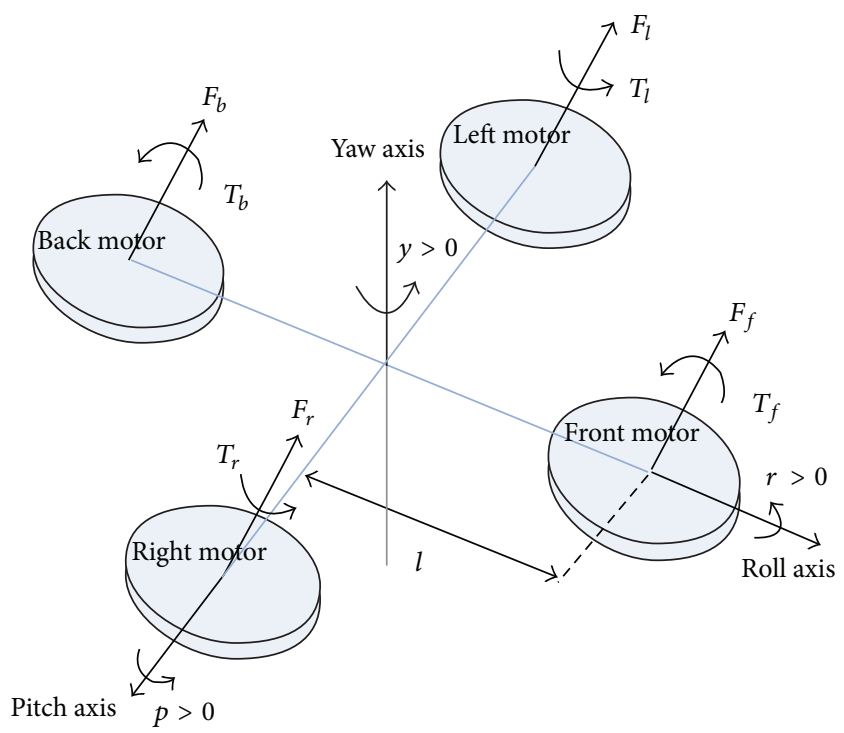

Figure 1: The structure schematic of quadrotor helicopter.

Under these assumptions, using the Newton-Euler Equation, the dynamics equations are written in the following way:

$$
\begin{gathered}
m \ddot{\xi}=F_{L}+F_{D}+G, \\
J \dot{\Omega}=-\Omega \times J \Omega+\Gamma_{f}-\Gamma_{a}-\Gamma_{g},
\end{gathered}
$$

where $\xi=(x, y, z) \in R^{3}$ is the position of the centre of mass with respect to the inertial frame, $m$ is the total mass of this structure, and $J \in R^{3 \times 3}$ is a constant inertia matrix of quadrotor with respect to the body fixed frame. That is,

$$
J=\left(\begin{array}{ccc}
J_{\phi} & 0 & 0 \\
0 & J_{\theta} & 0 \\
0 & 0 & J_{\psi}
\end{array}\right)
$$

where $J_{\phi}, J_{\theta}$, and $J_{\psi}$ represent rotational inertia of the roll axis, pitch axis, and yaw axis, respectively. $\Omega$ represents the angular velocity of quadrotor expressed in the body fixed frame such as

$$
\Omega=\left(\begin{array}{ccc}
1 & 0 & -\sin \theta \\
0 & \cos \phi & \cos \theta \sin \phi \\
0 & -\sin \phi & \cos \phi \cos \theta
\end{array}\right)\left(\begin{array}{c}
\dot{\phi} \\
\dot{\theta} \\
\dot{\varphi}
\end{array}\right)
$$

where $\phi, \theta$, and $\varphi$ are roll, pitch, and yaw angles, respectively. When the attitude angles are small, $\Omega=(p, q, r) \approx$ $(\dot{\phi}, \dot{\theta}, \dot{\varphi})$.

There are some details about all terms in (1) and (2) below.

In (1), firstly, $F_{L}$ represents the total lift generated by the four rotors and the expression is

$$
F_{L}=\left(\begin{array}{c}
\cos \phi \sin \theta \cos \psi+\sin \phi \sin \psi \\
\cos \phi \sin \theta \sin \psi-\sin \phi \cos \psi \\
\cos \phi \cos \theta
\end{array}\right)\left(F_{r}+F_{l}+F_{f}+F_{b}\right),
$$

where $F_{r}=K_{p} \omega_{r}^{2}, F_{l}=K_{p} \omega_{l}^{2}, F_{f}=K_{p} \omega_{f}^{2}$, and $F_{b}=K_{p} \omega_{b}^{2}$, which are the lift generated by right rotor, left one, front one, 


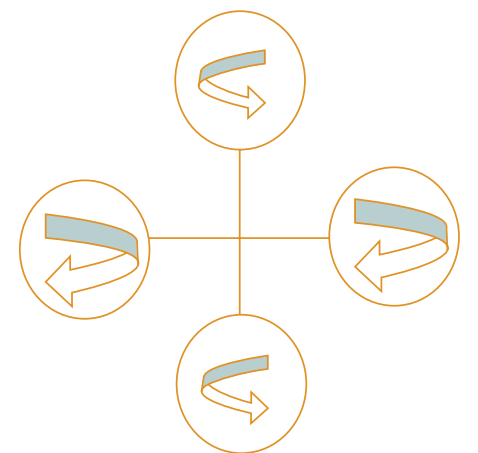

Yaw motion

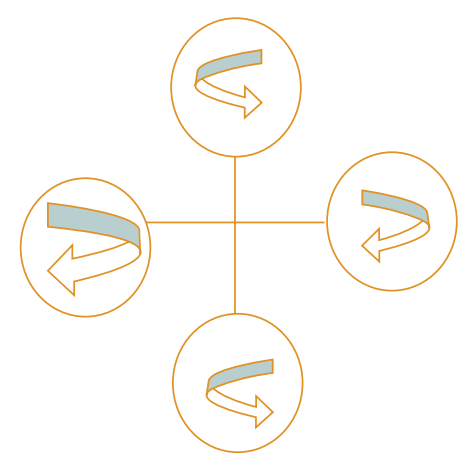

Roll motion

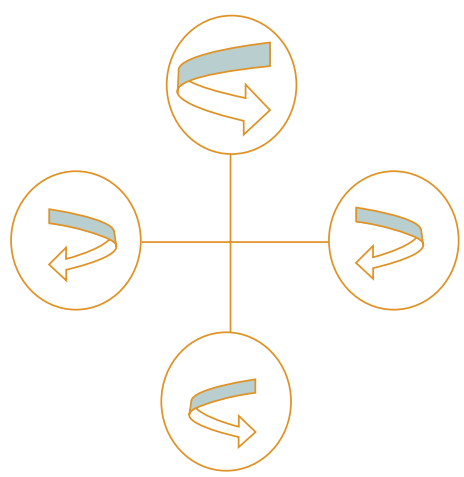

Pitch motion

FIGURE 2: The attitude control principle of quadrotor helicopter.

and back one, respectively. Then $K_{p}$ is the lift coefficient, and $\omega_{r}, \omega_{l}, \omega_{f}$, and $\omega_{b}$ represent the rotation speed of right rotor, left one, front one, and back one, respectively.

Secondly, $F_{D}$, the total drag, can be described by

$$
F_{D}=\left(\begin{array}{ccc}
-K_{f d x} & 0 & 0 \\
0 & -K_{f d y} & 0 \\
0 & 0 & -K_{f d z}
\end{array}\right) \dot{\xi},
$$

where $-K_{f d x},-K_{f d y}$, and $-K_{f d z}$ are the component of drag coefficient in $x, y$, and $z$ axis, respectively.

Then, $G$ is the gravity, which is

$$
G=\left(\begin{array}{c}
0 \\
0 \\
-m g
\end{array}\right) .
$$

In (2), firstly, $\Gamma_{f}$, the moment of lift, when decomposed into each axis of the body fixed frame, can be described by

$$
\Gamma_{f}=\left(\begin{array}{c}
l\left(F_{r}-F_{l}\right) \\
l\left(F_{f}-F_{b}\right) \\
C_{D}\left(\omega_{l}^{2}+\omega_{r}^{2}-\omega_{b}^{2}-\omega_{f}^{2}\right)
\end{array}\right),
$$

where $l$ is the distance between the axis of any rotor and the centre of mass and $C_{D}$ is the coefficient of drag moment.

Secondly, $\Gamma_{a}$ represents the total pneumatic friction torque, whose expression is

$$
\Gamma_{a}=\left(\begin{array}{ccc}
K_{\mathrm{fax}} & 0 & 0 \\
0 & K_{\mathrm{fay}} & 0 \\
0 & 0 & K_{\mathrm{faz}}
\end{array}\right)\|\Omega\|^{2},
$$

where $K_{\text {fax }}, K_{\text {fay }}$, and $K_{\text {faz }}$ are the component of pneumatic friction coefficient in $x, y$, and $z$ axis, respectively.

Next, $\Gamma_{g}$ is the resultant moment under gyroscopic effect and can be defined as

$$
\Gamma_{g}=\Omega \times J_{r}\left(\begin{array}{c}
0 \\
\frac{0}{\Omega}
\end{array}\right),
$$

where $J_{r}$ is the moment of inertia of the rotor; moreover, $\bar{\Omega}=$ $\omega_{l}+\omega_{r}-\omega_{b}-\omega_{f}$.
In conclusion, the dynamic model of quadrotor helicopter can be written as

$$
\begin{aligned}
& \ddot{\phi}=\frac{1}{J_{\phi}}\left[\left(J_{\theta}-J_{\psi}\right) \dot{\psi} \dot{\theta}-K_{\mathrm{fax}} \dot{\phi}^{2}-J_{r} \bar{\Omega} \dot{\theta}+l\left(F_{r}-F_{l}\right)\right], \\
& \ddot{\theta}=\frac{1}{J_{\theta}}\left[\left(J_{\psi}-J_{\phi}\right) \dot{\psi} \dot{\phi}-K_{\mathrm{fay}} \dot{\theta}^{2}+J_{r} \bar{\Omega} \dot{\phi}+l\left(F_{f}-F_{b}\right)\right], \\
& \ddot{\psi}=\frac{1}{J_{\psi}}\left[\left(J_{\phi}-J_{\theta}\right) \dot{\phi} \dot{\theta}-K_{\mathrm{faz}} \dot{\psi}^{2}+\frac{C_{D}}{K_{p}}\left(F_{r}+F_{l}-F_{f}-F_{b}\right)\right],
\end{aligned}
$$

where $F_{r}, F_{f}, F_{l}$, and $F_{b}$ defined previously are the inputs of this system.

To simplify the representations, we define

$$
\begin{aligned}
\left(\begin{array}{c}
U_{\phi} \\
U_{\theta} \\
U_{\psi}
\end{array}\right) & =\left(\begin{array}{cccc}
1 & 0 & -1 & 0 \\
0 & 1 & 0 & -1 \\
\frac{C_{D}}{K_{P}} & -\frac{C_{D}}{K_{P}} & \frac{C_{D}}{K_{P}} & -\frac{C_{D}}{K_{P}}
\end{array}\right)\left(\begin{array}{c}
F_{r} \\
F_{f} \\
F_{l} \\
F_{b}
\end{array}\right) \\
& =L\left(\begin{array}{c}
F_{r} \\
F_{f} \\
F_{l} \\
F_{b}
\end{array}\right) .
\end{aligned}
$$

Due to the limit of the power of each electromotor, there exists a maximum rotation speed $\omega_{\max }$ for each rotor. It is assumed that each $\omega_{\max }$ is equal because of the property of symmetry in the quadrotor helicopter. Therefore, the inputs meet the following conditions:

$$
\begin{gathered}
-K_{p} \omega_{\max }^{2} \leq U_{\phi} \leq K_{p} \omega_{\max }^{2}, \\
-K_{p} \omega_{\max }^{2} \leq U_{\theta} \leq K_{p} \omega_{\max }^{2}, \\
-2 C_{D} \omega_{\max }^{2} \leq U_{\psi} \leq 2 C_{D} \omega_{\max }^{2} .
\end{gathered}
$$


TABLE 1: Fault mode.

\begin{tabular}{lc}
\hline Fault parameter & State of system \\
\hline$\lambda_{i}=1, \sigma_{i}=0$ & Normal \\
$0<\lambda_{i}<1, \sigma_{i}=0$ & Loss of effectiveness \\
$\lambda_{i}=0, \sigma_{i}=1$ & Lock-in-place \\
\hline
\end{tabular}

In addition, the dynamic of the DC-electromotor which drives rotors is shown below:

$$
\begin{gathered}
V=R I+L \frac{d I}{d t}+K_{e} \omega, \\
K_{m} I=J_{r} \frac{d \omega}{d t}+K_{r} \omega^{2}+C_{s},
\end{gathered}
$$

where $R$ is the internal resistance of electromotor and $K_{e}, K_{m}$, and $K_{r}$ are the electric torque constant, mechanical torque constant, and load constant torque, respectively. Then, $C_{s}$ denotes the solid friction.

Based on this analysis, let $x=[\phi, \dot{\phi}, \theta, \dot{\theta}, \psi, \dot{\psi}]^{T} \in R^{6}$, $u=\left[F_{r}, F_{f}, F_{l}, F_{b}\right]^{T}=\left[u_{1}, u_{2}, u_{3}, u_{4}\right]^{T} \in R^{4}$ be the state and the control input vectors, respectively. The state equation of this system can be written in the following affine nonlinear representation:

$$
\dot{x}=f(x)+g(x) u
$$

where

$$
\begin{gathered}
f(x)=\left(\begin{array}{c}
x_{2} \\
a_{1} x_{4} x_{6}+a_{2} x_{2}^{2}+a_{3} \bar{\Omega} x_{4} \\
x_{4} \\
a_{4} x_{2} x_{6}+a_{5} x_{4}^{2}+a_{6} \bar{\Omega} x_{2} \\
x_{6} \\
a_{7} x_{2} x_{4}+a_{8} x_{6}^{2}
\end{array}\right), \\
g(x)=\left(\begin{array}{cccc}
0 & 0 & 0 & 0 \\
b_{1} & 0 & -b_{1} & 0 \\
0 & 0 & 0 & 0 \\
0 & b_{2} & 0 & -b_{2} \\
0 & 0 & 0 & 0 \\
b_{3} & -b_{3} & b_{3} & -b_{3}
\end{array}\right)
\end{gathered}
$$

with

$$
\begin{gathered}
a_{1}=\frac{J_{\theta}-J_{\psi}}{J_{\phi}}, \quad a_{2}=-\frac{K_{\mathrm{fax}}}{J_{\phi}}, \quad a_{3}=-\frac{J_{r}}{J_{\phi}}, \\
a_{4}=\frac{J_{\psi}-J_{\phi}}{J_{\theta}}, \quad a_{5}=-\frac{K_{\mathrm{fay}}}{J_{\theta}}, \quad a_{6}=\frac{J_{r}}{J_{\theta}}, \\
a_{7}=\frac{J_{\phi}-J_{\theta}}{J_{\psi}}, \quad a_{8}=-\frac{K_{\mathrm{faz}}}{J_{\psi}}, \\
b_{1}=\frac{l}{J_{\phi}}, \quad b_{2}=\frac{l}{J_{\theta}}, \quad b_{3}=\frac{C_{D}}{J_{\psi} K_{p}} .
\end{gathered}
$$

2.2. Actuator Fault Model. According to report of research, the actuator of the helicopter can be easily damaged. In view of quadrotor helicopter, when the actuator fault occurs, the rotation speed of rotors will be abruptly changed so that the attitude system of quadrotor will vary rapidly or even lose control.

In this paper, we consider actuator faults including loss of effectiveness and lock-in-place. When any actuator has failed, we can denote a general actuator fault model as [9]

$$
u=\lambda \underline{u}+\sigma(\bar{u}-\lambda \underline{u})
$$

where $\lambda=\operatorname{diag}\left[\lambda_{1}, \lambda_{2}, \lambda_{3}, \lambda_{4}\right], v=\left[v_{1}, v_{2}, v_{3}, v_{4}\right]^{T}, \sigma=$ $\operatorname{diag}\left[\sigma_{1}, \sigma_{2}, \sigma_{3}, \sigma_{4}\right]$, and $\bar{u}=\left[\bar{u}_{1}, \bar{u}_{2}, \bar{u}_{3}, \bar{u}_{4}\right]^{T} . \lambda_{i}$ denotes the percentage of the remaining effective part of the corresponding actuator. $\underline{u}_{i}$ denotes the applied control vector, while $\sigma_{i}\left(\sigma_{i}=0\right.$ or 1$)$ is used to describe the lock-in-place fault. If $\lambda_{i}=1$ and $\sigma_{i}=0$, no fault occurs in this actuator. When $0<\lambda_{i}<1$ and $\sigma_{i}=0$, the corresponding actuator loses partial effectiveness. The case of $\lambda_{i}=0, \sigma_{i}=1$ means that the actuator is stuck at some unknown place where $\bar{u}_{i}$ is the constant value. The considered faults can be synthesized by Table 1.

Inspired from [10], we can define another input vector $v=$ $\left[U_{\phi}, U_{\theta}, U_{\psi}\right]^{T} \in R^{3}$ so that the number of the output which will be given in the next section is equal to that of the input.

Thus, we design the control vector $v$ instead of $\underline{u}$. However, the applied input $\underline{u}$ can be achieved by

$$
\underline{u}=L^{-} v
$$

where $L^{-}=\left(L^{T} L\right)^{-1} L^{T}$ is the generalized inverse matrix of $L$.

To attain the control objective, we propose to use a proportional actuator structure as follows [11]:

$$
v=c v_{0},
$$

where $c=\operatorname{diag}\left[c_{1}, c_{2}, c_{3}\right]$ represents the proportional actuation gain matrix and $v_{0}=\left[v_{01}, v_{02}, v_{03}\right]^{T}$ is adaptive fuzzy controller that we proposed. by

Using (18), (19), and (20), the system (15) can be described

$$
\dot{x}=f^{\prime}(x)+g^{\prime}(x) v_{0},
$$

where $f^{\prime}(x)=f(x)+g(x) \sigma \bar{u}, g^{\prime}(x)=g(x)(I-\sigma) \lambda L^{-} c$.

\section{Direct Self-Repairing Control Strategy}

The direct adaptive fuzzy control based on sliding mode is proposed to actualize self-repairing control in this paper. Adaptive fuzzy sliding mode control combines the advantages between adaptive fuzzy control and sliding mode control, which can not only adjust the adaptation law on line when uncertain function exists but also ensure the robustness of the considered nonlinear system.

The control block diagram is shown in Figure 3.

We consider the output of this quadrotor helicopter attitude system as $y=[\phi, \theta, \psi]^{T} \in R^{3}$. 


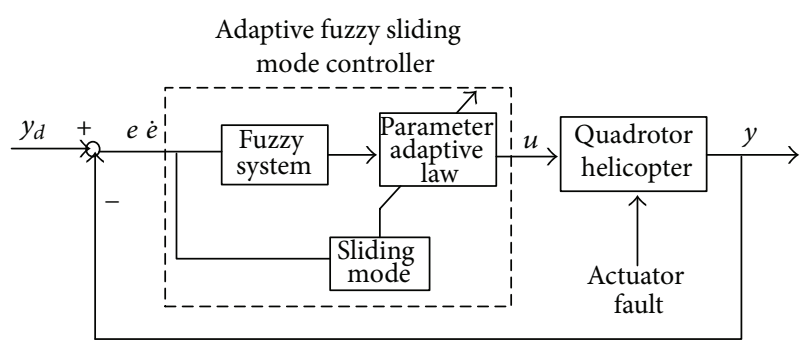

FIGURE 3: The control block diagram for adaptive fuzzy sliding mode control.

Then, the dynamic equation of the output can be rewritten in the following form:

$$
\begin{aligned}
y^{(2)} & =F(x)+G(x) v_{0} \\
& =\left[\begin{array}{llllll}
0 & 1 & 0 & 0 & 0 & 0 \\
0 & 0 & 0 & 1 & 0 & 0 \\
0 & 0 & 0 & 0 & 0 & 1
\end{array}\right]\left[f^{\prime}(x)+g^{\prime}(x) v_{0}\right] .
\end{aligned}
$$

Our task is to design a robust adaptive fuzzy controller based on sliding mode. There is no couple between each output vector, so we can consider the sliding surface in the state error space as

$$
s=e+k \dot{e},
$$

where $s=\left[s_{1}, s_{2}, s_{3}\right]^{T}$ and $k=\operatorname{diag}\left[k_{1}, k_{2}, k_{3}\right]\left(k_{i}>0 i=\right.$ $1,2,3) e=\left[e_{1}, e_{2}, e_{3}\right]^{T}$ with

$$
\begin{aligned}
& e_{1}(t)=\phi_{d}-\phi, \\
& e_{2}(t)=\theta_{d}-\theta, \\
& e_{3}(t)=\psi_{d}-\psi
\end{aligned}
$$

with the desired output signal denoted by $y_{d}=\left[\phi_{d}, \theta_{d}, \psi_{d}\right]^{T}$.

The time derivative of (23) can be written as

$$
\dot{s}=\alpha-k\left[F(x)+G(x) \nu_{0}\right],
$$

where $\alpha$ is given as follows:

$$
\alpha=\left[k_{1} \ddot{\phi}_{d}+\dot{e}_{1}, k_{2} \ddot{\theta}_{d}+\dot{e}_{2}, k_{3} \ddot{\theta}_{d}+\dot{e}_{3}\right]^{T} .
$$

If the functions $F(x)$ and $G(x)$ are known, to achieve the control objective, one can use the following ideal nonlinear control law:

$$
v_{0}^{*}=G^{-1}(x)\left(-F(x)+k^{-1} \alpha+\frac{s}{\gamma^{2}}\right) .
$$

Effectively, when we select the control input as $\nu_{0}=\nu_{0}^{*}$, (25) simplifies to

$$
\dot{s}=-k \frac{s}{\gamma^{2}}
$$

Here we design a Lyapunov function as $V=(1 / 2) s^{T} s \geq 0$. Then we have $\dot{V}=\dot{s}^{T} s=-\left(1 / \gamma^{2}\right) s^{T} k s \leq 0$, which indicates that the sliding mode defined can be achieved.

Thus, we can conclude that $s(t) \rightarrow 0$ as $t \rightarrow \infty$; therefore, $e(t)$ converges to zero, and the whole system is stable.
3.1. Adaptive Fuzzy Control Law. However, when the actuators of quadrotor helicopter have faults, the functions $F(x)$ and $G(x)$ are unknown so that the ideal controller designed previously cannot be used.

To overcome this problem, we propose to use an adaptive fuzzy system to approximate this ideal control law. Moreover, the parameters of this fuzzy controller are updated by the error between the fuzzy controller and the desired one.

According to the approximation theorem [12], there exists an optimal input based on fuzzy control approximating uniformly the ideal control law (27) such that

$$
v_{0}=\xi(x) \theta
$$

Then the fuzzy approximation error is

$$
\varepsilon=v_{0}^{*}-v_{0}=v_{0}^{*}-\xi(x) \theta,
$$

where $\theta=\left[\theta_{1}, \theta_{2}, \theta_{3}\right]^{T}$ is an unknown parameter vector which minimizes the approximation error on a compact set $\Omega$. And $\xi(x)=\operatorname{diag}\left[\xi_{1}(x), \xi_{2}(x), \xi_{3}(x)\right]$ is the matrix of fuzzy basis function suitably selected.

In this paper, we assume that the fuzzy controller proposed satisfies the universal approximation property over the compact set $\Omega$, which is assumed to be large enough so that the state variables remain inside it under closed-loop control. Therefore, it is reasonable to assume that the fuzzy approximation error is bounded for all $x \in \Omega$.

3.2. Parameter Adaptation Law. In the preview, we recall that the parameter vector $\theta$ is unknown. So the parameter estimate $\widehat{\theta}$ based on a gradient descent adaptation algorithm will be developed in this subsection.

To design a suitable adaptation law, our goal is to minimize the approximation error between $v_{0}^{*}$ and $v_{0}$.

Hence, the parameter estimate $\widehat{\theta}$ is obtained according to the following theorem.

Theorem 1. The adaptive fuzzy control law

$$
\widehat{\nu}_{0}=\xi(x) \hat{\theta}
$$

equipped with the following adaption law:

$$
\dot{\hat{\theta}}=\eta \xi(x)\left(\dot{s}+k \frac{s}{\gamma^{2}}\right)-\eta \tau \widehat{\theta},
$$

where $\eta, \tau$ are positive constant parameters, guarantees the stability and the robustness of the system with actuator failures (18).

Proof. Firstly, the estimate error $\widetilde{\theta}$ should be defined by

$$
\widetilde{\theta}=\theta-\widehat{\theta} .
$$

Then, we substitute the proposed control law (31) to (25), and the derivative of sliding surface changes to be

$$
\dot{s}=-k \frac{s}{\gamma^{2}}+G(x)\left(v_{0}^{*}-\widehat{\nu}_{0}\right)=-k \frac{s}{\gamma^{2}}+G(x) \varepsilon^{\prime},
$$


where $\varepsilon^{\prime}=\varepsilon+\xi(x) \widetilde{\theta}$, denoted to be the error between desired input and actual input.

Then, another Lyapunov function is designed by the following form:

$$
V^{\prime}=\frac{1}{2} s^{T} s+\frac{1}{2 \eta} \widetilde{\theta}^{T} \widetilde{\theta} .
$$

Obviously,

$$
\dot{V}^{\prime}=s^{T} \dot{s}+\frac{1}{\eta} \widetilde{\theta}^{T} \dot{\tilde{\theta}}=s^{T} \dot{s}-\frac{1}{\eta} \widetilde{\theta}^{T} \dot{\hat{\theta}} .
$$

Substituting (32) and (34), we can get

$$
\begin{aligned}
\dot{V}^{\prime} & =-\frac{1}{\gamma^{2}} s^{T} k s+s^{T} G(x) \varepsilon^{\prime}-\widetilde{\theta}^{T} \xi G(x) \varepsilon^{\prime}+\tau \widetilde{\theta}^{T} \hat{\theta} \\
& =-\frac{1}{\gamma^{2}} s^{T} k s+s^{T} G(x) \varepsilon^{\prime}-(\xi \widetilde{\theta})^{T} G(x) \varepsilon^{\prime}+\tau \widetilde{\theta}^{T} \hat{\theta} \\
& =-\frac{1}{\gamma^{2}} s^{T} k s+s^{T} G(x) \varepsilon^{\prime}-\varepsilon^{\prime T} G(x) \varepsilon^{\prime}+\varepsilon^{T} G(x) \varepsilon^{\prime}+\tau \widetilde{\theta}^{T} \hat{\theta}
\end{aligned}
$$

using the inequalities

$$
\begin{aligned}
\widetilde{\theta}^{T} \widehat{\theta}= & -\frac{1}{2}\|\widetilde{\theta}\|^{2}-\frac{1}{2}\|\widehat{\theta}\|^{2}+\frac{1}{2}\|\widetilde{\theta}+\widehat{\theta}\|^{2} \\
\leq- & \frac{1}{2}\|\widetilde{\theta}\|^{2}+\frac{1}{2}\|\theta\|^{2}, \\
s^{T} G(x) \varepsilon^{\prime}= & \left.-\left(\frac{1}{2} \varepsilon^{\prime}+s\right)\right)^{T} G(x)\left(\frac{1}{2} \varepsilon^{\prime}+s\right) \\
& +\frac{1}{4} \varepsilon^{\prime T} G(x) \varepsilon^{\prime}+s^{T} G(x) s \\
\leq & \frac{1}{4} \varepsilon^{\prime T} G(x) \varepsilon^{\prime}+s^{T} G(x) s, \\
\varepsilon^{T} G(x) \varepsilon^{\prime} \leq & \frac{1}{4} \varepsilon^{\prime T} G(x) \varepsilon^{\prime}+\varepsilon^{T} G(x) \varepsilon .
\end{aligned}
$$

Equation (37) can be bounded as

$$
\begin{aligned}
\dot{V}^{\prime} \leq & -\frac{1}{\gamma^{2}} s^{T} k s-\frac{\tau}{2}\|\widetilde{\theta}\|^{2}-\frac{1}{2} \varepsilon^{\prime T} G(x) \varepsilon^{\prime}+\varepsilon^{T} G(x) \varepsilon \\
& +s^{T} G(x) s+\frac{\tau}{2}\|\theta\|^{2} .
\end{aligned}
$$

Using the fact that the control gain matrix is positive definite, so there exists a positive constant $\beta$ such that $G(x) \leq \beta I$; then, let another positive constant denote $k_{0}=$ $\min \left\{k_{1}, k_{2}, k_{3}\right\}$ so that $k \geq k_{0} I$; moreover, we select $\tau=$ $2\left(\left(k_{0} / \gamma^{2}\right)-\beta\right)$ to be positive, and this leads to

$$
\begin{aligned}
\dot{V}^{\prime} \leq & -\frac{\tau}{2}\left(s^{T} s+\widetilde{\theta}^{T} \tilde{\theta}\right)-\frac{1}{2} \varepsilon^{{ }^{T}} G(x) \varepsilon^{\prime} \\
& +\beta \varepsilon^{T} \varepsilon+\frac{\tau}{2}\|\theta\|^{2} \\
\leq & -\frac{\tau}{2} V^{\prime}+\beta \varepsilon^{T} \varepsilon+\frac{\tau}{2}\|\theta\|^{2} .
\end{aligned}
$$

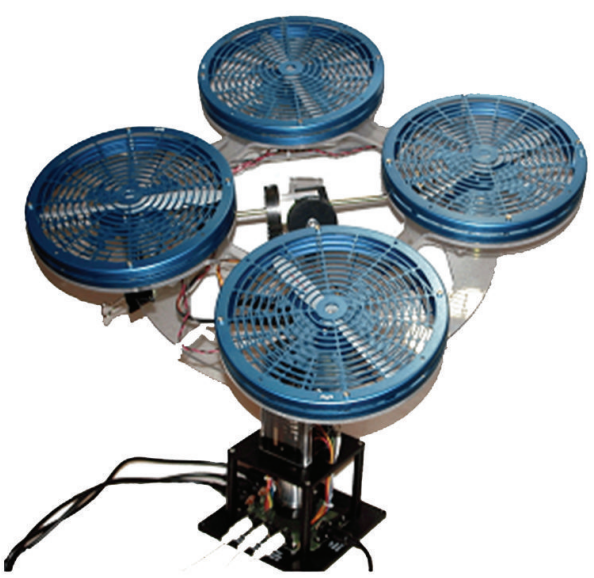

FiguRE 4: 3-DOF hover experimental platform.

Since the desired parameter vector $\theta$ is a constant vector, $\varepsilon$ is assumed to be bounded. Also we can define a positive constant bound $\beta_{0}$ as

$$
\beta_{0}=\sup \left(\frac{\tau}{2}\|\theta\|^{2}+\beta \varepsilon^{T} \varepsilon\right)
$$

Then the inequality of $\dot{V}^{\prime}$ is simplified to

$$
\dot{V}^{\prime} \leq-\frac{\tau}{2} V^{\prime}+\beta_{0}
$$

By quoting a theorem in [10], we can prove that the parameter error vector $\widetilde{\theta}$ and the tracking error vector $e(t)$ are bounded. Furthermore, due to the bound of $\dot{e}(t)$, we can also conclude that the state vector $x$ is bounded as well. Therefore, this adaptive fuzzy control system based on sliding mode for quadrotor helicopter is stable and has good performance of tracking.

\section{Simulation Results}

In this paper, we take the 3-DOF hover helicopter shown in Figure 4 which is produced by Quanser Company as the research object to simulate the operation of quadrotor helicopter attitude system. The 3-DOF hover helicopter consists of electric motors, rotors, helicopter body, power-supply module, encoders (sensors), and so forth. In the existing software platform, we can design the direct adaptive fuzzy sliding mode control system under the circumstance of MATLAB REAL-TIME. With the help of Quanser's supporting software, the block diagrams of MATLAB Simulink can be directly encoded into $C$ language which downloads to realtime simulation system from supporting PIC card through the parallel port [13]. After that, we can do the simulation experiment to verify the practicability of the control method proposed. 


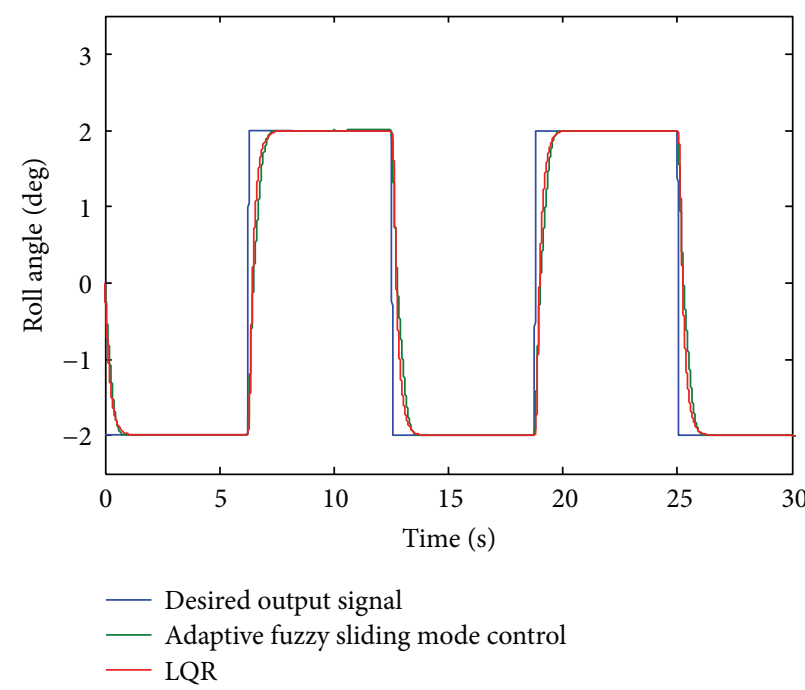

(a) Rolling angle tracking curves

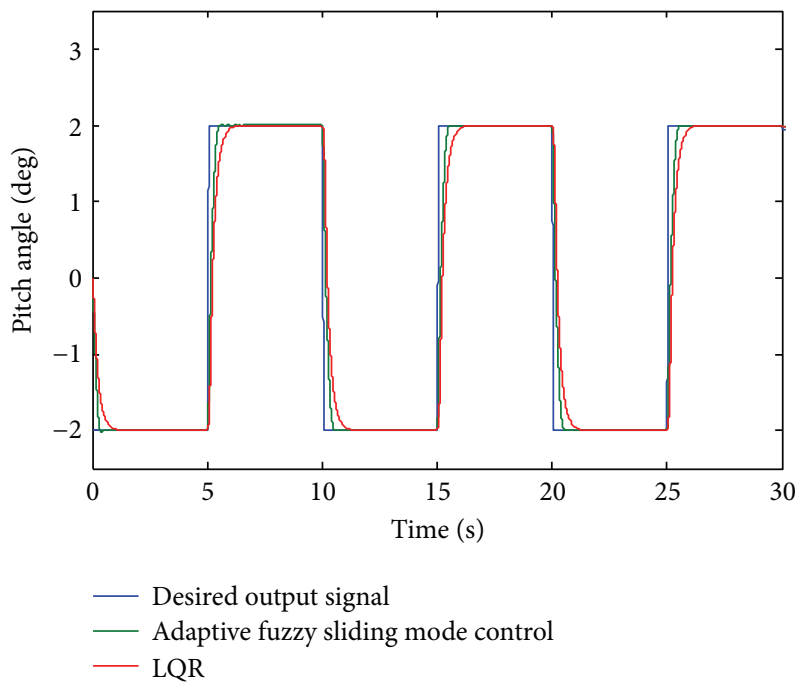

(b) Pitching angle tracking curves

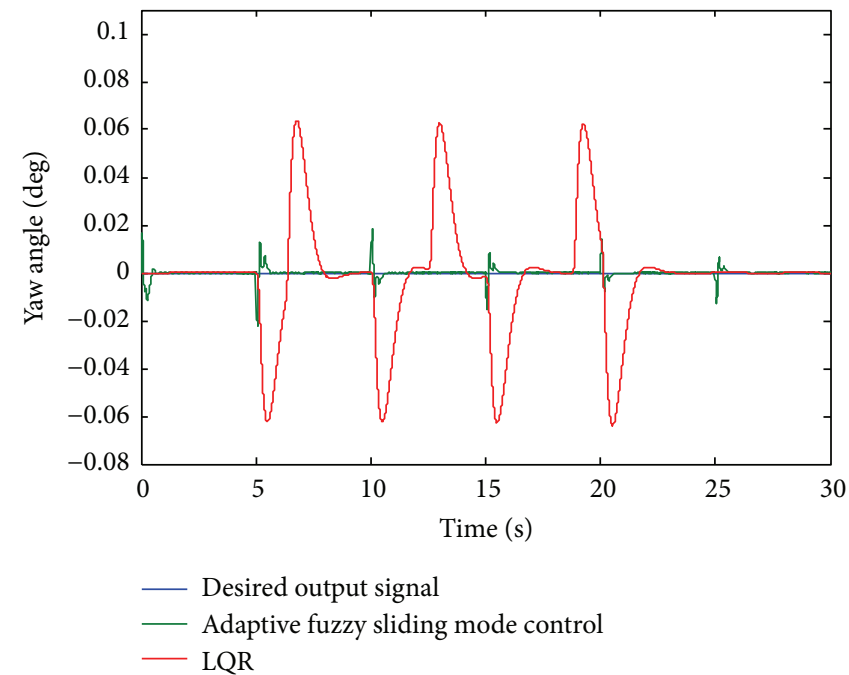

(c) Yawing angle tracking curves

FIGURE 5: The output response curves with no fault.

According to the user's guide of Quanser Hover, the system parameters are given by

$$
\begin{gathered}
K_{\mathrm{fax}}=0.0080, \quad K_{\mathrm{fay}}=0.0080, \quad K_{\mathrm{faz}}=0.0091, \\
J_{\phi}=0.0552, \quad J_{\theta}=0.0552, \quad J_{\varphi}=0.11, \\
l=0.197, \quad J_{r}=0.1188, \quad K_{p}=0.0036, \quad C_{D}=0.0036 .
\end{gathered}
$$

Furthermore, to illustrate the superiority of the method proposed, system dynamic performance under the adaptive fuzzy sliding mode control will be compared with that under the LQR method when these three cases occur: normal, the loss of effectiveness of actuators, and lock-in-place fault.

The design procedure of LQR is shown below.

Firstly, the affine nonlinear model of quadrotor helicopter should be linearized. Next, we can select suitable weight matrices $Q, R$, where $Q=\operatorname{diag}([125,250,250,0,10,10])$, $R=0.01 \cdot \operatorname{diag}([1,1,1])$. The control matrix $K$ is achieved by using LQR commands in MATLAB, which is

$$
K=\left[\begin{array}{cccccc}
0 & 0 & 158.1139 & 0 & 0 & 41.7939 \\
0 & 158.1139 & 0 & 0 & 41.7939 & 0 \\
122.4745 & 0 & 0 & 86.6881 & 0 & 0
\end{array}\right]
$$

Hence, the LQR controller is described by $u=K\left(x-x_{d}\right)$, where $x_{d}=\left[\phi_{d}, 0, \theta_{d}, 0, \varphi_{d}, 0\right]^{T}$ is the desired state vector.

In all simulation cases, the desired rolling angle, pitching angle, and yawing angle are selected to be square wave with the amplitude $2^{\circ}$ and square wave with the amplitudes $2^{\circ}$ and $0^{\circ}$, respectively.

Three fuzzy systems in the form of (29) are used to generate the control signals $u_{1}, u_{2}$, and $u_{3}$. Each system has the input as $z_{1}=\left[e_{1}(t), \dot{e}_{1}(t)\right]^{T}, z_{2}=\left[e_{2}(t), \dot{e}_{2}(t)\right]^{T}$, 


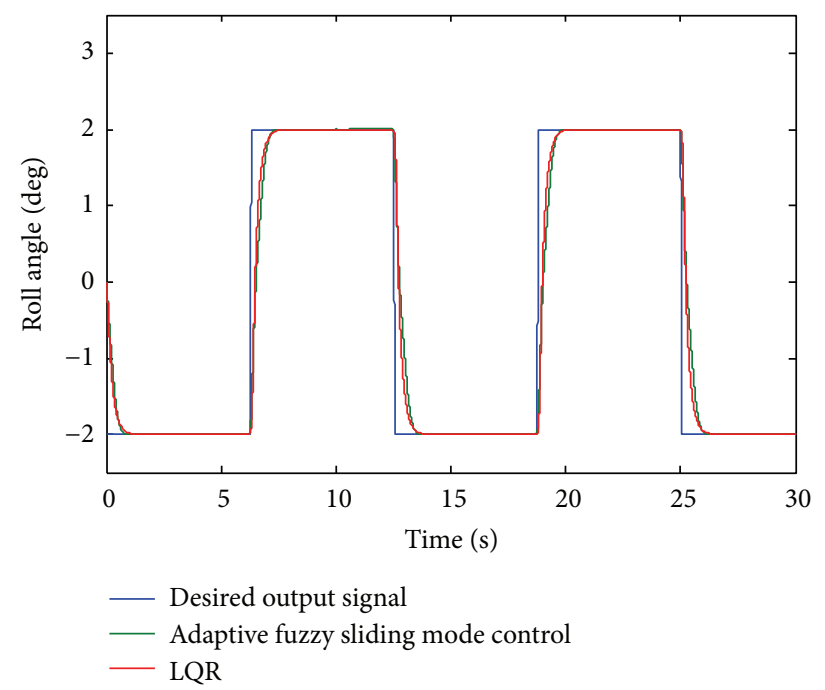

(a) Rolling angle tracking curves

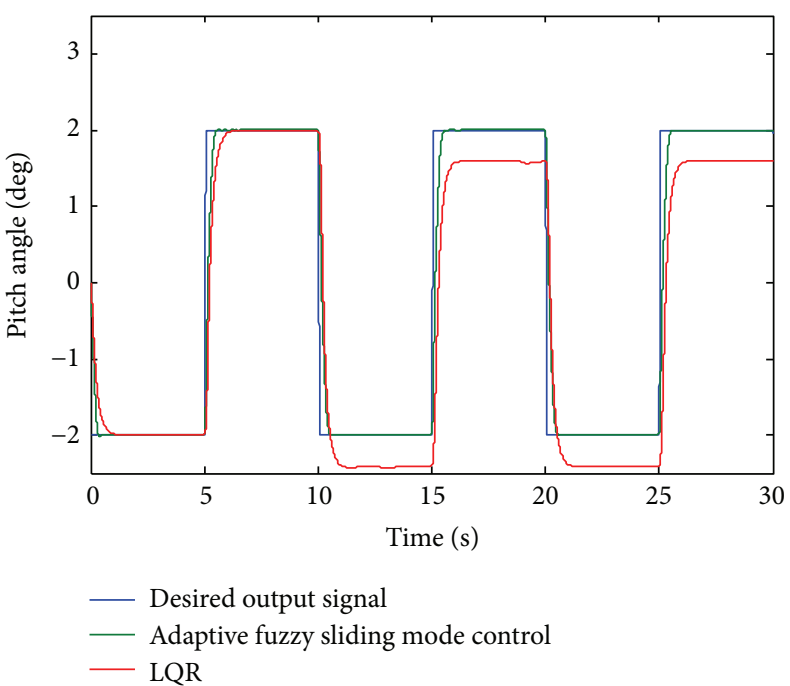

(b) Pitching angle tracking curves

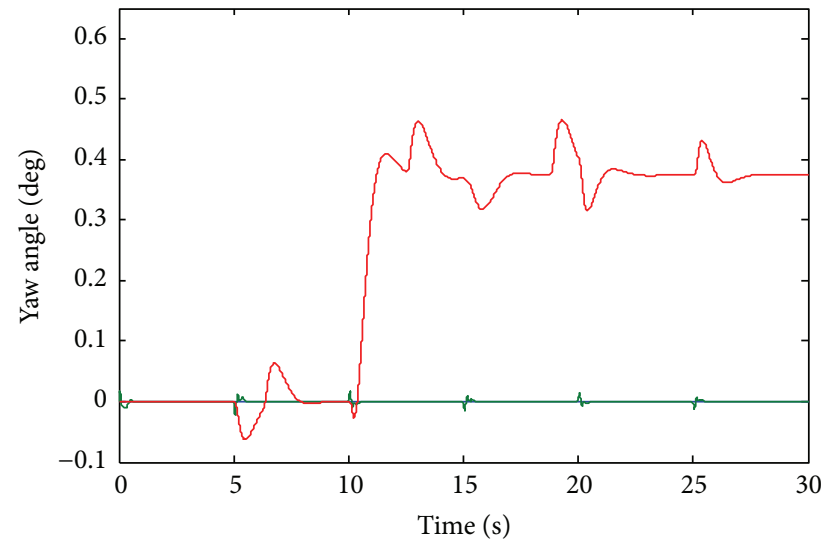

Desired output signal
— Adaptive fuzzy sliding mode control
_ LQR

(c) Yawing angle tracking curves

FIGURE 6: The output response curves with the loss of effectiveness in $u_{2}$.

and $z_{3}=\left[e_{3}(t), \dot{e}_{3}(t)\right]^{T}$, respectively. For each input variable in $z_{1}, z_{2}$, and $z_{3}$, seven Gaussian membership functions which give 49 fuzzy rules are defined as

$$
\begin{aligned}
& \mu_{F_{1}}\left(z_{i j}\right)=\exp \left(-\frac{1}{2}\left(\frac{z_{i j}+1}{0.14}\right)^{2}\right), \\
& \mu_{F_{2}}\left(z_{i j}\right)=\exp \left(-\frac{1}{2}\left(\frac{z_{i j}+0.67}{0.14}\right)^{2}\right), \\
& \mu_{F_{3}}\left(z_{i j}\right)=\exp \left(-\frac{1}{2}\left(\frac{z_{i j}+0.33}{0.14}\right)^{2}\right), \\
& \mu_{F_{4}}\left(z_{i j}\right)=\exp \left(-\frac{1}{2}\left(\frac{z_{i j}}{0.14}\right)^{2}\right),
\end{aligned}
$$

$$
\begin{aligned}
& \mu_{F_{5}}\left(z_{i j}\right)=\exp \left(-\frac{1}{2}\left(\frac{z_{i j}-0.33}{0.14}\right)^{2}\right) \\
& \mu_{F_{6}}\left(z_{i j}\right)=\exp \left(-\frac{1}{2}\left(\frac{z_{i j}-0.67}{0.14}\right)^{2}\right) \\
& \mu_{F_{7}}\left(z_{i j}\right)=\exp \left(-\frac{1}{2}\left(\frac{z_{i j}-1}{0.14}\right)^{2}\right)
\end{aligned}
$$

where $i=1,2, j=1,2$.

In addition, the time of simulation and step size are set to be $30 \mathrm{~s}$ and $0.001 \mathrm{~s}$, respectively. The other design parameters used in this simulation are chosen as follows:

$$
k=\operatorname{diag}[10,10,10], \quad \gamma=1, \quad \tau=0.001, \quad \eta=20 .
$$




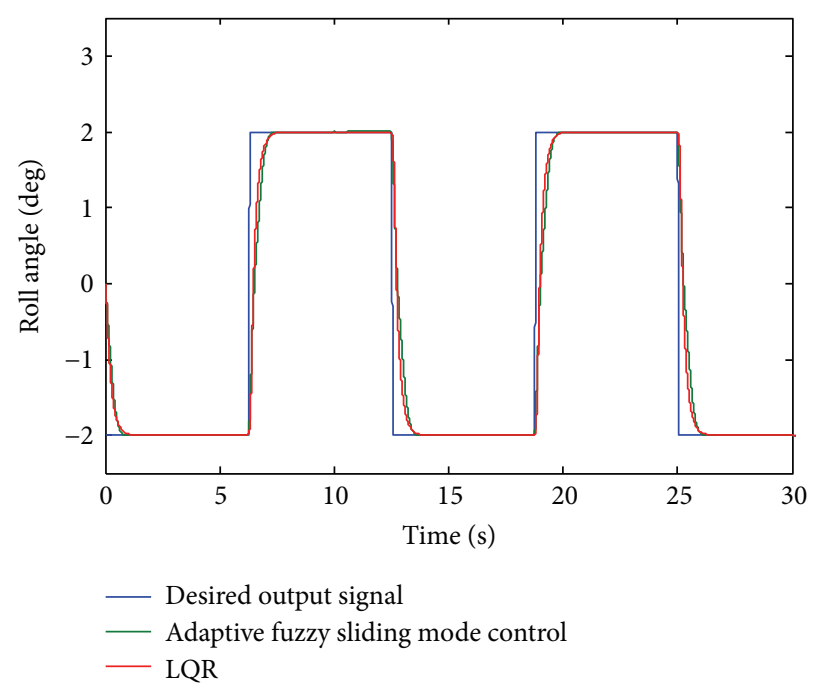

(a) Rolling angle tracking curves

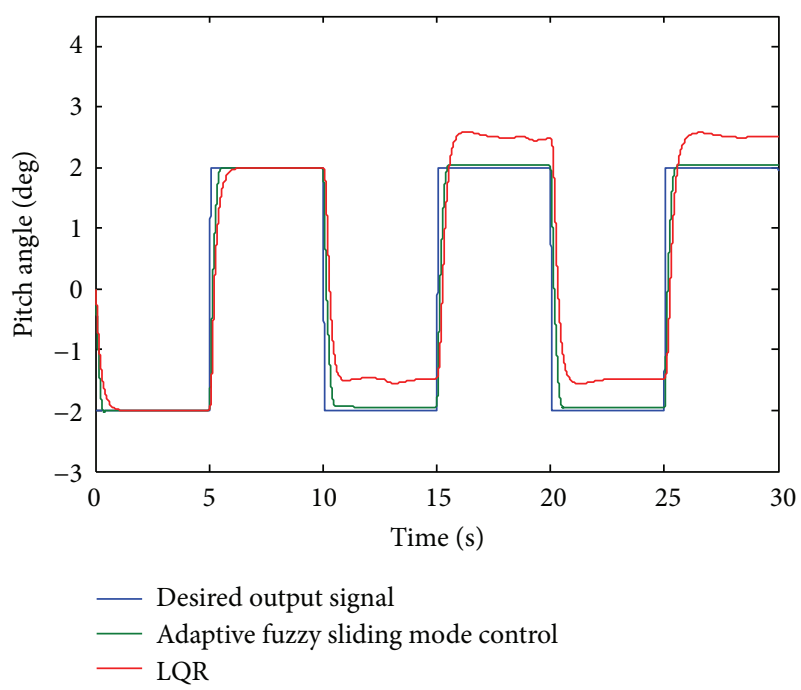

(b) Pitching angle tracking curves

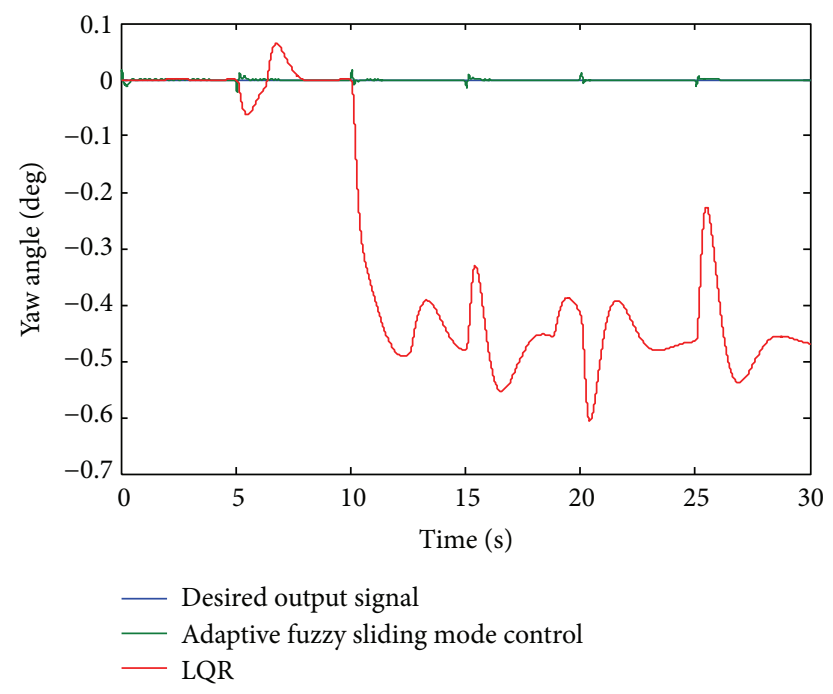

(c) Yawing angle tracking curves

FIgURE 7: The output response curves with the lock-in-place in $u_{2}$.

(1) When no fault happened in this system, the simulation curves on adaptive fuzzy sliding mode controller and LQR controller are shown in Figure 5.

From Figure 5, it can be seen that the system can track the desired output well with the help of both controllers and has good dynamic performance. However, as for yawing angle, under the adaptive fuzzy controller based on sliding mode, the system can track signal almost without error while LQR controller cannot achieve that, which demonstrates that the proposed adaptive fuzzy controller has a better tracking performance than LQR controller when the system is healthy.

(2) We suppose that the system is subject to loss of effectiveness at $10 \mathrm{~s}$ in input $u_{2}$, and this may lead to the changes of pitching angle and yawing angle. The rest of the simulation settings are the same. Thus, we can get the simulation curves under adaptive fuzzy sliding mode controller and LQR controller shown in Figure 6.

Refer to Figure 6; the conclusion is that, when the loss of effectiveness occurred in $u_{2}$, the system nearly has no influence and still tracks the signal very well with the help of adaptive fuzzy sliding mode controller. But the performance of LQR controller falls with the obvious tracking errors. So the superiority of adaptive fuzzy controller is proven again.

(3) Suppose that a lock-in-place fault occurs in $u_{2}$ at $10 \mathrm{~s}$. The rest of the simulation settings are unchanged. The simulation curves under adaptive fuzzy sliding mode controller and LQR controller are given in Figure 7.

We can obtain the same conclusion from Figure 7.

Without the loss of generality, another case is set to indicate that adaptive fuzzy sliding mode controller still 


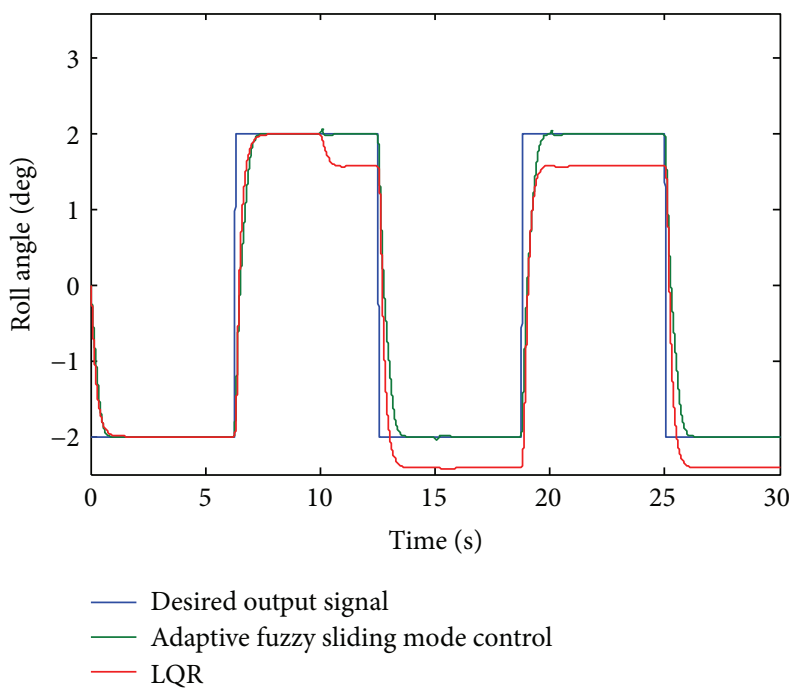

(a) Rolling angle tracking curves

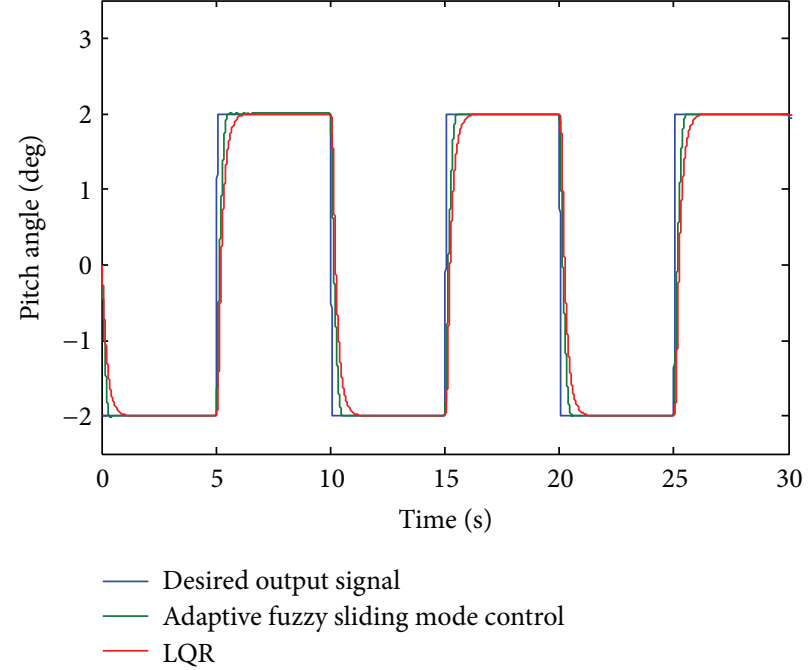

(b) Pitching angle tracking curves

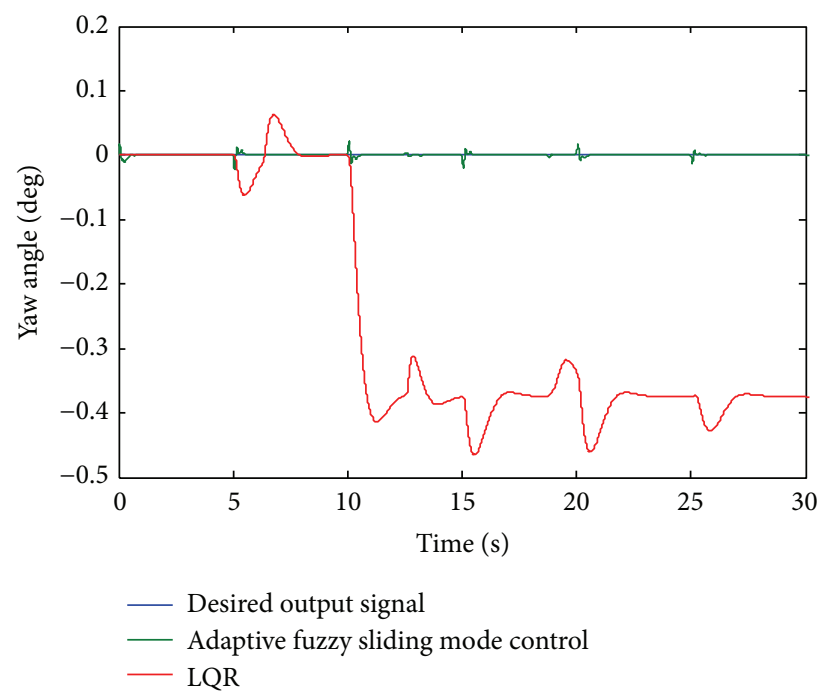

(c) Yawing angle tracking curves

FIgURE 8: The output response curves with the loss of effectiveness in $u_{1}$.

works and is better than LQR controller when the actuator fault occurs in other inputs.

(4) We suppose that the system loses effectiveness at $10 \mathrm{~s}$ in input $u_{1}$. The rest of the simulation settings are unchanged. Then the simulation curves under both controllers are given in Figure 8.

Remark. Compared with the related work in $[6,13]$, the good features of this paper are in 3 aspects: (1) fault diagnosis is not needed in the proposed method so that it can be easier to be applied to the engineering practice; (2) even without the process of fault diagnosis, the proposed control system can still deal with a variety of actuator faults such as loss of effectiveness and lock-in-place; (3) the dynamic performance of the attitude system based on adaptive fuzzy sliding mode control when fault occurs is more smooth.
To sum up, when the actuator faults such as loss of effectiveness and lock-in-place occur in the attitude system of quadrotor helicopter, under the adaptive fuzzy sliding mode controller, this system can still track the desired output signal very well and return to the normal performance very rapidly, which implies that the whole system has the certain capability of self-repairing.

\section{Conclusion}

In this paper, firstly, we built the affine nonlinear model for the quadrotor helicopter attitude system, which is MIMO. With the consideration of unknown actuator faults such as loss of effectiveness and lock-in-place, an adaptive fuzzy controller based on sliding mode has been proposed to realize the direct self-repairing control for this attitude system. Through a series of simulations, it has verified the availability 
of the proposed method which can make the system recover from the actuator faults and has good tracking performance.

\section{Conflict of Interests}

The authors declare that there is no conflict of interests regarding the publication of this paper.

\section{Acknowledgments}

This work is supported by the National Nature Science Foundation of China under Grants 61273171 and 61304112, Natural Science Foundation of Jiangsu Province (BK20131364), the Fundamental Research Funds for the Central Universities (no. NE2014202), and the Foundation of Graduate Innovation Center in NUAA (kfji130109).

\section{References}

[1] B. Nie, H. Ma, J. Wang, and J. Wang, "Study on actualities and critical technologies of micro/mini quadrotor," Electronics Optics and Control, vol. 14, no. 6, pp. 113-1117, 2007.

[2] S. Bouabdallah, A. Noth, and R. Siegwart, "PID versus LQ control techniques applied to an indoor micro Quadrotor," in Proceedings of the IEEE/RSJ International Conference on Intelligent Robots and Systems (IROS '04), pp. 2451-2456, IEEE, New York, NY, USA, October 2004.

[3] E. Altug, Vision Based Control of Unmanned Aerial Vehicles with Applications to autonomous Four Rotor Helicopter Quadrotor, University of Pennsylvania, Philadelphia, Pa, USA, 2003.

[4] S. Wang, Research of Quadrotor Control, Harbin Institute of Technology, Harbin, China, 2006.

[5] C. Yang, Z. Yang, X. Huang, and D. Xu, "Distributed Faulttolerant control for Quadrotor," Journal of Applied Sciences, vol. 31, no. 3, pp. 321-330, 2013.

[6] X. Gong, C. Peng, and Y. Tian, "Reliable attitude stability control of Quad-rotor based on fault-tolerant approach," in Proceedings of the IEEE International Conference on Mechatronics and Automation, pp. 1015-1020, IEEE, New York, NY, USA, 2012.

[7] F. Sharifi, M. Mirzaei, B. W. Gordon, and Y. Zhang, "Fault tolerant control of a quadrotor UAV using sliding mode control," in Proceedings of the 1st Conference on Control and Fault-Tolerant Systems (SysTol '10), pp. 239-244, New Jersey, NJ, USA, October 2010.

[8] C. Pedro, L. Rogelio, and E. D. Alejandro, Modeling and Control of Mini-Flying Machines, Springer, New York, NY, USA, 2005.

[9] X. Tang, G. Tao, and S. M. Joshi, "Compensation of nonlinear MIMO systems for uncertain actuator failures with an application to aircraft control," in Proceedings of the 41st IEEE Conference on Decision and Control, pp. 1245-1250, New York, NY, USA, December 2002.

[10] S. Labiod and T. M. Guerra, "Direct adaptive fuzzy control for a class of MIMO nonlinear systems," International Journal of Systems Science, vol. 38, no. 8, pp. 665-675, 2007.
[11] P. Li and G.-H. Yang, "Fault tolerant control for unknown nonlinear systems with actuator failures: an adaptive fuzzy approach," in Proceedings of the American Control Conference (ACC '08), pp. 4330-4335, usa, June 2008.

[12] L. Wang, Adaptive Fuzzy Systems Ad Control Design and Stability Analysis, Prentice Hall, Englewood Cliffs, NJ, USA, 1994.

[13] Quanser Consultiing, 3D Hover System, Quanser inc, 2005. 


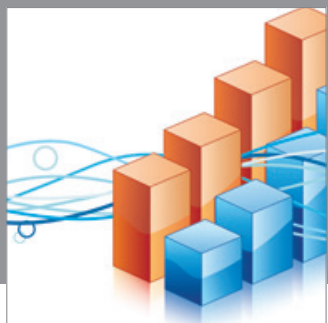

Advances in

Operations Research

mansans

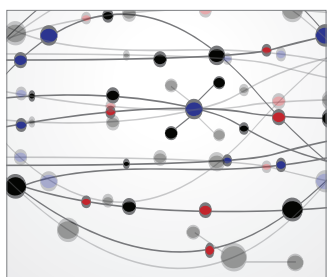

The Scientific World Journal
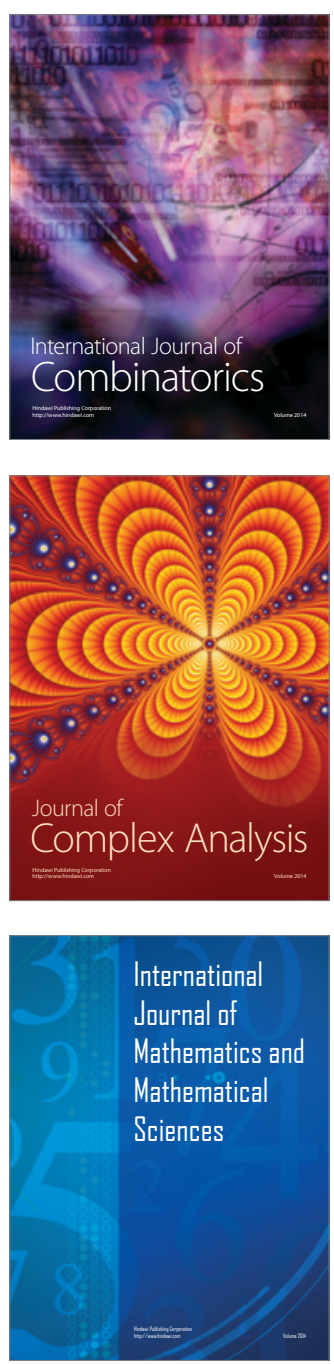
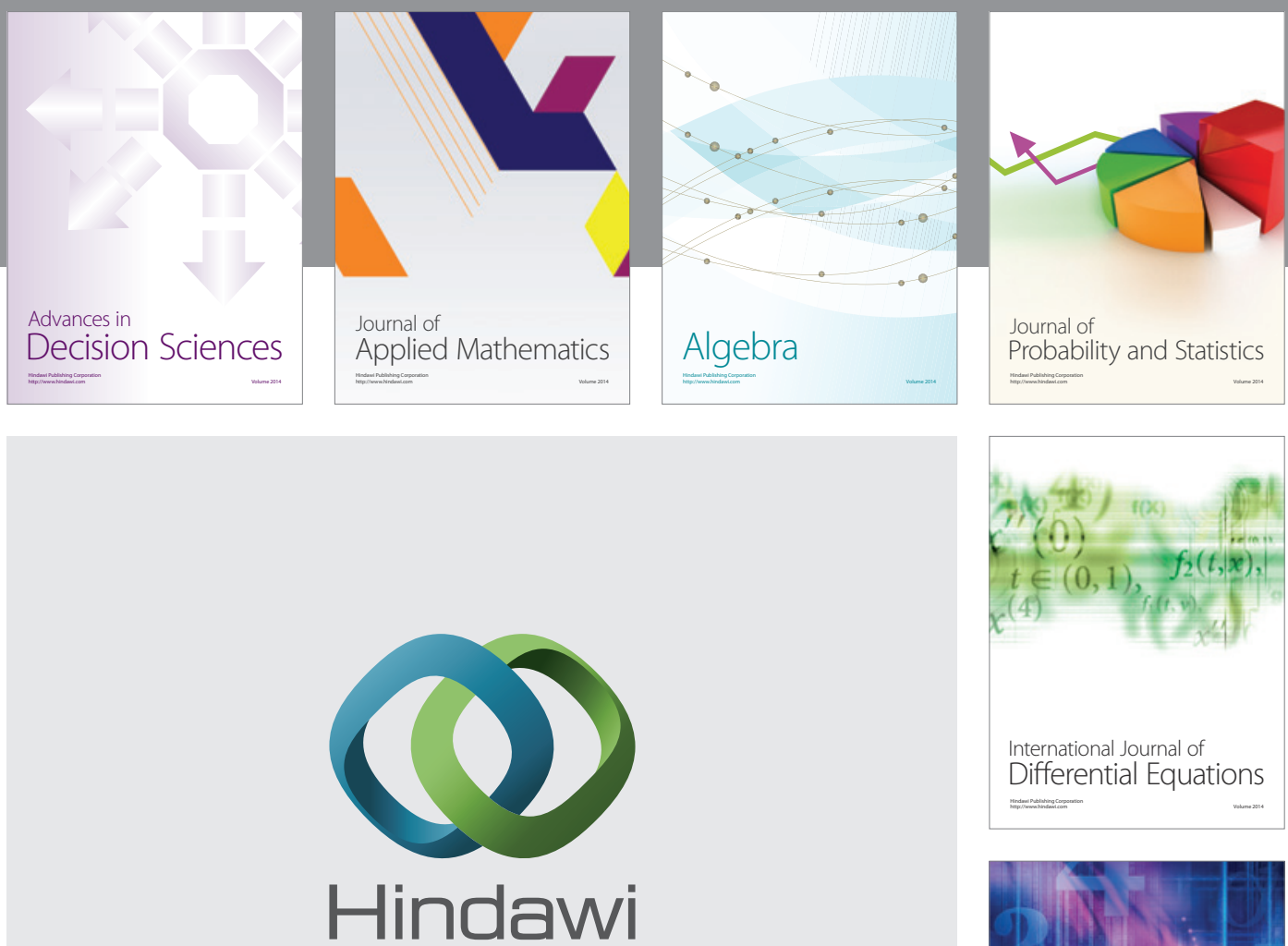

Submit your manuscripts at http://www.hindawi.com
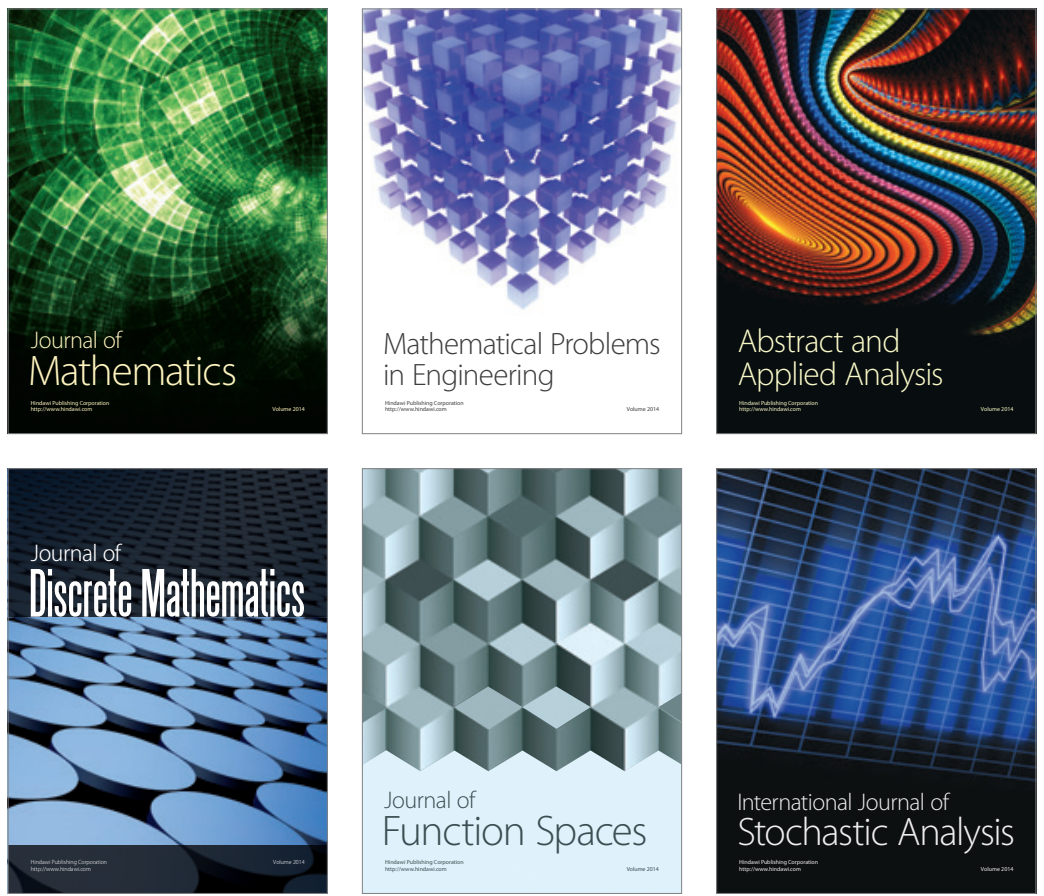

Journal of

Function Spaces

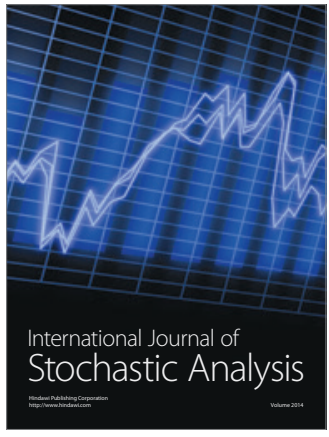

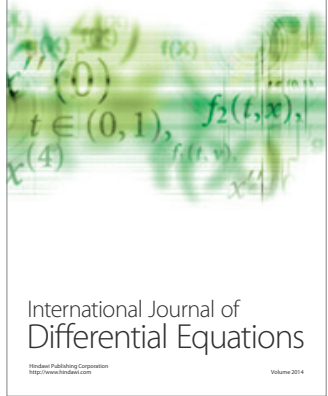
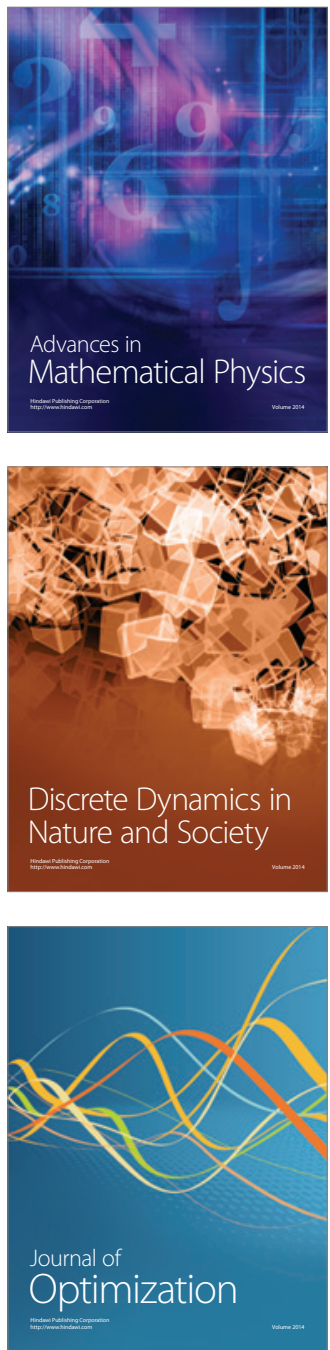